\title{
Reminiscences of the First Olfactory Neuroimaging Study on the 20th Anniversary of its Publication
}

\author{
Marilyn Jones-Gotman • Robert J. Zatorre
}

Received: 9 January 2012 / Accepted: 11 January 2012 / Published online: 28 January 2012

(C) Springer Science+Business Media, LLC 2012

In the 1980s, only a handful of publications existed on neural correlates of olfaction in humans. Psychophysical and behavioral papers were more numerous, and anatomical and physiological data derived from studies carried out in "macrosmic species", especially rats, existed, but the means with which to study brain function in humans was limited. We had the good fortune to have those means available to us. Working at the Montreal Neurological Institute, we had access to people with focal surgical excisions that had been performed as a treatment for intractable epileptic seizures. We each had ongoing research programs studying performance on various auditory, verbal, and visuospatial cognitive tasks as a function of surgical lesions in different brain regions. This paradigm allowed us to infer a significant role on the part of specific brain regions on particular tasks based on impaired performance when those regions were damaged. Neither of us specialized in olfaction at the time. Robert Zatorre's (RZ's) primary interest was in audition, and Marilyn Jones-Gotman's (MJG's) was memory, especially nonverbal memory. Curiosity about memory for odors led MJG to begin olfactory investigations, while his interest in perception was RZ's motivation. Being each poised independently and simultaneously to launch olfactory studies in the MNI patients, we decided to join forces.

We started without funding, and accumulating odorants to begin our experiments was an adventure. We acquired some in the hospital pharmacy and local grocery stores, but the more exciting acquisition came from the Brown University Smell Library, which was being closed because Trygg Engen, a

\footnotetext{
M. Jones-Gotman $(\bowtie) \cdot R$. J. Zatorre

Montreal, QC, Canada

e-mail: marilyn.jonesgotman@mcgill.ca

R. J. Zatorre

e-mail: robert.zatorre@mcgill.ca
}

longtime olfactory psychophysicist at Brown, was retiring. Because RZ had trained at Brown, he knew Trygg and his former student, Bob Mair, who kindly alerted us to this opportunity. We were given a fairly large number of odorants, which RZ transported in the trunk of his car from Brown to Montreal without being suspected by the border police or their dogs.

We started with the basics: detection thresholds, quality discrimination, memory, and identification. The unilateral surgical lesions of most of our patients were in the temporal lobes and also in the frontal lobes, with a few in parietal or occipital lobes. We found that although detection thresholds were not affected by any of these surgical lesions, there were deficits on all other tasks. The biggest deficits occurred specifically with orbitofrontal lesions and not with damage to other frontal cortical areas. There were impairments also after temporal lobe surgeries. Parietal or occipital lobe lesions did not affect our tasks. These results also usually showed a right hemispheric predominance in olfaction. The temporal and frontal lobe findings confirmed our predictions, based on findings in other species, that important olfactory functions depended on cortical structures in the anteromedial frontal cortex and in the orbitofrontal cortex. A laterality difference was a novel finding, not having been observed in animal studies, but because it replicated over several studies we were tempted to believe it to be real.

After working on olfaction for about 5 years with this lesion approach, we were very fortunate to have the opportunity to perform the first functional neuroimaging study of olfaction. In the late 1980s, there had been developments, at the MNI and elsewhere, in using positron emission tomography (PET) to study brain metabolism. Initial attempts using fluorodeoxyglucose as a tracer were largely unsuccessful because of the long acquisition times ( $>60 \mathrm{~min})$ and correspondingly poor ability to resolve different events or to test 
different conditions. But around the turn of the decade of the 1990s, a new technique was developed involving oxygen-15 water as the tracer, which permitted activation studies an order of magnitude faster $(60 \mathrm{~s})$ than the older techniques. Coupled with the idea coming out of the St. Louis PET imaging group to perform voxel-by-voxel subtraction analyses, a new concept at the time, we now had the tools to perform an almost unlimited set of experiments. We jumped on the possibility to explore olfaction, excited to see whether any of the findings from the patient studies would hold up in healthy humans with this new PET method to study cerebral blood flow.

Our first PET experiment was pretty basic: we presented eight different odors birhinally every $10 \mathrm{~s}$, asking only that our volunteers look straight ahead and inhale when in their peripheral vision they saw the odorant approach their nose. We also instructed them to sniff in exactly the same way every time, and during the control condition, we told them that there would be no odor but again to sniff in exactly the same way as before. We worked together, one of us keeping the timing accurate with a stopwatch and the other leaning in to waft each odorant under a subject's nostrils. We presented the odorants on cotton swabs that were attached to small glass wands. These methods may seem primitive (and they are) compared to today's sophistication, but using them and comparing the two conditions-sniffing odorants compared to sniffing "blanks"-we demonstrated localized brain activity during smelling of odors. The findings were exciting because for the first time we could actually visualize the olfactory cortices in vivo in humans. The greatest activations were observed bilaterally in what we determined had to be piriform cortex, at the junction of the temporal and inferior frontal cortices, and in lateral orbitofrontal cortex, with predominance for the right side. These results fit well with our previous lesion data. They fit also with predictions made by Tanake, based on experiments in other primates, of the presumed location of human olfactory regions. In retrospect, we were lucky: had we used only one odorant instead of eight there would likely have been adaptation that would have reduced or eliminated the piriform activation. If we had not told the subjects that there would be no odor during the control scan, they might have searched for it, reducing the difference between the experimental and control conditions, as subsequent studies have suggested.

We wrote the report and submitted it to Nature, but to our disappointment, it was rejected without review. However, our colleague, Alan Evans, was undeterred: he told us to send the paper back and to attach a color photo of the brain image - quite a novelty in 1991 - to the front of the letter (this was back in the days when submitting a paper actually meant that a piece of paper was involved!). Our letter also explained further what we thought was the significance of our findings. The editors came to their senses, and to our great pleasure, the paper was accepted after the usual review. It was published in 1992-20 years ago already — with Alan Evans and the late Ernst Meyer as coauthors.

We are pleased to note that in most basic respects, the initial findings appear to have held up well. Of course, more careful and systematic findings have since been carried out, and the development of functional MRI a few years after PET allowed much more precise questions to be asked. But this first PET paper opened the door for us and for many others to begin exploring different aspects of olfaction and related themes. Over time, RZ worked less in olfaction as he developed a major program of research in the cognitive neuroscience of auditory processing, while MJG divided her time between olfactory studies and research related to epilepsy. She was fortunate to attract a number of gifted students who were able to contribute to the new field of odor neuroimaging, and RZ continued to participate in the studies generated by those students. These included Dana Small, who expanded our research from olfaction to include gustation, Lauren Dade, who explored aspects of olfactory memory and learning, and Jelena Djordjevic, who studied mental imagery of the chemical senses. Most recently, Johan Lundström and Johannes Frasnelli worked as postdoctoral fellows with MJG, investigating perception of biologically significant odors. Several of these students have continued to work in the chemical senses and have made important contributions on their own.

The field has grown immensely since those early days. The methods and questions are much more sophisticated. The field is accumulating knowledge about how different aspects of smell and taste are represented in the brain, and how the sense of smell impacts human life. Although our research interests have largely migrated elsewhere at this point, we maintain an active interest in seeing where the once-fledgling field of chemical senses neuroimaging is going and are pleased to have contributed to it near its beginning. 